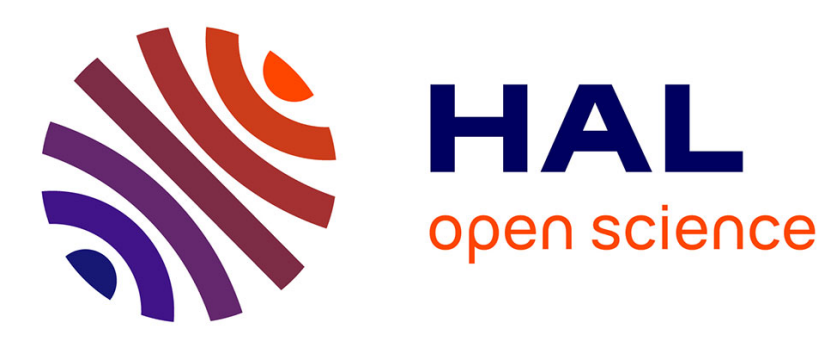

\title{
The ultrafast high-peak power lasers in future biomedical and medical X-ray imaging
}

Jean-Claude Kieffer, S C Fourmaux, A Krol

\section{To cite this version:}

Jean-Claude Kieffer, S C Fourmaux, A Krol. The ultrafast high-peak power lasers in future biomedical and medical X-ray imaging. 19th International Conference and School on Quantum Electronics, Alexandros Serafetinides; Sanka Gateva; Latchevar Avromov, Sep 2016, Sozopol, Bulgaria. pp.1022612-1, 10.1117/12.2261795 . hal-01441646

\section{HAL Id: hal-01441646 \\ https://hal.science/hal-01441646}

Submitted on 20 Jan 2017

HAL is a multi-disciplinary open access archive for the deposit and dissemination of scientific research documents, whether they are published or not. The documents may come from teaching and research institutions in France or abroad, or from public or private research centers.
L'archive ouverte pluridisciplinaire HAL, est destinée au dépôt et à la diffusion de documents scientifiques de niveau recherche, publiés ou non, émanant des établissements d'enseignement et de recherche français ou étrangers, des laboratoires publics ou privés. 


\title{
The ultrafast high-peak power lasers in future biomedical and medical $X$-ray imaging
}

\author{
J.C. Kieffer ${ }^{1}$, S. Fourmaux ${ }^{1}$, A. Krol $^{2}$ \\ ${ }^{1}$ INRS-EMT, Varennes, Québec, Canada \\ ${ }^{2}$ Upstate Medical University, SUNY, Syracuse, USA
}

\begin{abstract}
This paper reviews recent progresses in ultrafast laser-based $X$-ray sources and their potential applications to high throughput X-ray imaging. Prospects for the utilization of $X$-rays sources related to the Laser Wakefield electron Acceleration (LWFA) are more specifically discussed with emphasis on application in diagnostic radiology.
\end{abstract}

\section{INTRODUCTION}

Lasers, invented in 1960, are currently extensively used in medicine for diagnosis and treatment of diseases, as well as patients (re)positioning, respiratory motion gating, oximetry and countless other applications. Lasers are also found myriad applications in biomedical research in applications ranging from fluorescence microscopy [1] to microdissection [2] and in many others. Laser light with wavelengths between ultraviolet (UV) and infrared (IR) parts of spectrum allows faster, less invasive and safer clinical protocols with better outcome in many medical disciplines including dermatology, ophthalmology, dentistry, otolaryngology, urology, cardiology, neurosurgery and orthopedics [3]. The clinical and preclinical X-ray imaging [4] is based on conventional X-ray tube technology initiated in 1895. Even though, it has been demonstrated that lasers can provide bright secondary sources of X-rays, such laser-based Xray sources have not been introduced to medicine. We discussed fourteen years ago [5] the potential impact of the ultrafast laser-based X-ray sources on medical imaging, with a particular emphasis on the use of K-alpha radiation for low-dose high-resolution mammography and non-invasive coronary artery angiography.

Since that time remarkable progress has been made worldwide in the field of conventional (X-ray tube based) medical $\mathrm{X}$-ray imaging. Despite this progress, there is still a need for imaging with significantly higher spatial resolution, better soft tissue contrast resolution, lower radiation dose, and shorter scan duration than presently possible. The conventional medical X-ray tube is mature technology that after over hundred years of evolution reached its technological limits and has obvious advantages including relatively small form factor, high reliability and reasonable cost. Nonetheless, it also has numerous inherent deficiencies including a relatively large effective X-ray spot size and a limited control of the Xray spectral shape.

The field of ultrafast lasers technology and the field of high-brilliance and high-power laser-based X-ray sources is undergoing rapid development but this technology is still not ready for clinical trials for medical X-ray imaging applications. The commercial success of the future medical X-ray imaging systems integrating the ultrafast laser technology will depend mainly on the added clinical value, as compared with existing conventional technologies and the cost effectiveness. As the medical use of X-ray radiation in diagnostic radiology has grown exponentially in the recent years and as the imaging medical equipment is evolving rapidly, any new technology must undergo a careful assessment of the magnitude of exposure to ionizing radiation and potential risks $[6,7]$.

Advances in basic physics and laser technology including introduction of powerful ultrafast lasers (100TW - 1PW) suggest that in the near future it may be possible to built laser-based X-ray imaging devices that could overcome the limits of the conventional systems and shift X-ray imaging paradigms allowing low-dose, high-resolution and fast 
clinical and preclinical imaging. Two key types of laser-based X-ray sources, the K-alpha X-ray sources and the Laser Wakefield X-ray sources that have been developed over the recent years are major candidates for (bio)medical imaging with X-rays in the $15 \mathrm{keV}-150 \mathrm{keV}$ energy range. This article reviews critical issues pertinent to the application of these laser-based X-ray sources to (bio)medical imaging and discusses for the first time the possible impact of the novel Laser Wakefield electron Acceleration (LWEA) X-ray sources: the laser Betatron and Inverse Compton scattering X-ray sources

\section{PRESENT STATUS OF X-RAY BASED MEDICAL AND BIOMEDICAL IMAGING}

X-ray based medical imaging, backbone of radiology, includes many imaging and interventional modalities and is a result of continuous development for over 100 years [8]. Still, many challenges remain. For example, imaging of dense breast tissue in mammography is severely limited with reduced mammographic sensitivity and specificity and with the suboptimal dose utilization $[9,10]$. This is because the X-ray spectra produced by mammographic X-ray tube are suboptimal for this imaging task. Thus better control of spectral distribution of produced X-rays would be highly desirable. It is well accepted that higher spatial resolution, i.e. smaller X-ray focal spot, would be highly desirable in many X-ray imaging sub-modalities leading to better clinical exams specificity and sensitivity.

In the biomedical-imaging arena x-ray imaging plays critical and unique role providing structural but also, with the use of imaging probes, functional information. As an example, X-ray imaging, especially high-resolution micro-computed tomography (micro-CT), is emerging as a key tool to improve planting efficiency [11-13]. Increasing food production and efficiency is a fundamental world challenge for the XXI century. Plant performance in a crop context is determined by a multitude of internal and external factors; the response to this challenge includes an optimization of the crop production and crop improvements through breeding and through understanding the coupling between genetics and phenotyping [14].

Recent discoveries in genomics, and in molecular and cell biology have led to a wide use of small animal models of human disease. As a result, micro-CT has become an important tool in modern biomedical research [15]. Functional imaging of small animal models has been accomplished by introduction of various modalities, including micro-PET [16] and micro-SPECT [17], while anatomic imaging of soft tissue has been realized by means of magnetic resonance microscopy [18,19], micro-ultrasound [20,21] and micro-CT [22] with dual X-ray energy [23], with nanoparticles [24, 25], and with contrast media [26]. Synchrotron radiation has also been also used but it has been suffering from very high facility cost and limited user base [27]. Recently, multimodality micro-imaging, such as micro-PET/CT, micro-MR/PET, and micro-SPECT/CT, have been used to realize fusion of functional and anatomic information [28-31]. However, conventional micro-focal X-ray tube used in in vivo micro-CT with effective x-ray focal spot size in the 20-60 $\mu \mathrm{m}$ range can only be operated at relatively low power not exceeding $\sim 50 \mathrm{~W}$. It results in rather long micro-CT scan duration in the 10-60 min. range, depending on the desired spatial resolution.

X-ray phase-contrast (XPC) imaging has emerged recently giving rise to improved soft tissue density resolution in biomedical imaging. This imaging method is based on Denis Gabor holography principle [32,33]. The first XPC images were obtained in 1995 with synchrotron [34] and microfocal X-ray tube sources [35]. Since then, many groups have carried out phase contrast imaging with synchrotron and X-ray tube sources and in various geometries [36]. XPC imaging requires an X-ray source with a very small effective focal spot size, a high-resolution X-ray detector and an appropriate location of the sample and the detector in the beam path. If the detector is placed in proximity of the sample, a conventional absorption image is obtained. However, if the X-ray source is sufficiently small (i.e. it is spatially coherent) and the detector is placed far beyond the sample, a near field Fresnel diffraction patterns can be obtained. The images formed in these conditions contain information on both the real (X-ray phase-shift) and the imaginary (X-ray absorption) components of the X-ray refractive index in the object. Therefore, even if the differences in X-ray absorption between different types of tissue are too small to create sufficient image contrast in conventional $\mathrm{x}$-ray radiography and tomography, the method that allows X-ray phase-shift mapping might provide sufficient image contrast for differentiation of various types of tissue. The application of X-ray phase contrast imaging with X-ray tubes and synchrotrons has been widely discussed and includes mammography [37-40], tomography of biological tissues [41, 42], biomedical applications [43], and plant and soil science [44,45] among others. Phase contrast imaging shows considerable promise for medical, preclinical and life sciences X-ray imaging [46]. However, a substantial amount of 
work still remains to be done to determine the optimal X-ray source parameters, imaging system geometry and detector parameters, system cost and its clinical efficacy. The major obstacles preventing wide spread of XPC imaging are aforementioned deficiencies of X-ray tube technology: large effective focal spot size and low output power.

\section{ULTRAFAST LASER K-ALPHA BASED IMAGING}

The laser-produced plasma (LPP) X-ray source has been studied extensively especially in the context of X-ray lithography [47] and inertial confinement fusion (ICF) [48]. Continuous bremsstrahlung and characteristic X-ray emission result from the interaction at high optical power density $\left(10^{15}-10^{18} \mathrm{~W} / \mathrm{cm}^{2}\right)$ laser beam with solids, liquids or clusters. Application of these novel X-ray sources to medical imaging was proposed very early [49]. However, in order to generate optical power density sufficient to obtain the desired, for intended applications X-ray flux, previous designers have relied almost exclusively on large (or very large for ICF), energetic lasers generating laser light pulses with nanosecond duration. Focal spots created were large $(0.3-1.0 \mathrm{~mm})$, due to lateral transport in LPP produced by long pulses [50], i.e. they were comparable with conventional X-ray tubes.

The invention of chirped-pulse amplification (CPA) in the late 1980's allowed the achievement of very high optical power density $\left(10^{18}-10^{20} \mathrm{~W} / \mathrm{cm}^{2}\right)$ delivered to the target by the laser beam from relatively compact (table-top) Terawatt lasers with femtosecond pulse duration [51-53]. The development of the femtosecond laser technology has been particularly impressive since the last published review [54]. The ultrashort laser technology is very diverse, but advances in both laser architecture and materials (fibers, slabs and thin disks) allowed massive increase in the average and peak power [55-57]. High peak power lasers (above 100TW) are now commercially available and widely used to accelerate protons to the multi- $\mathrm{MeV}$ energy range [58-61], electrons to the $\mathrm{GeV}$ energy range [62-64], and to generate electromagnetic radiation spanning from $\mathrm{THz}$ to X-rays $[65,66]$. A comprehensive review of the status of Petawatt class lasers (above 200TW) worldwide recently published [67] shows how fast this field is expanding. Many new academic projects have emerged worldwide. They have been aiming at demonstrating the potential of ultrafast laser-based electromagnetic radiation sources and particle accelerators for medical and life sciences application [68].

\subsection{Ultrafast laser-based K-alpha $X$-ray sources}

A K-alpha $(\mathrm{K} \alpha) \mathrm{X}$-ray source is created during interaction between a high-power femtosecond laser and a high-Z solid target or liquid heavy metal jet. Such source is characterized by a small footprint, a narrow spectrum (K $\alpha$ characteristic radiation) with a tunable energy (obtained by changing the target material's elemental composition) in the $5-50 \mathrm{keV}$ energy range, and an effective X-ray source size (limited lateral transport with ultrafast laser pulses) in the tens of micrometers range [5,69-83]. We discussed the prospects for the utilization of such ultrafast laser produced plasmas Xray sources in diagnostic radiology [69] with a particular emphasis on application in non-invasive coronary artery angiography (X-rays around $35 \mathrm{keV}$ ) and low dose high-resolution mammography (X-rays in the 17-30 keV energy range). Our initial approach to solid targets $[5,70,71]$ was to investigate the emission of K-alpha radiation using second harmonic generation $(400 \mathrm{~nm})$ in order to produce a very high contrast ratio optical pulse and generate X-ray source size close to the laser focusing spot size (a few $\mu \mathrm{m}$ in diameter). One drawback of this approach is relatively low hot electron temperature generated at shorter wavelength resulting in lower conversion efficiency from laser optical energy into Kalpha X-rays energy (typically $2 \times 10^{-5}$ for Mo K-alpha line at $17 \mathrm{keV}$ ). Recently, we have been able to achieve conversion efficiencies of $2 \times 10^{-4}$ (for Mo K-alpha line at $\sim 17.4 \mathrm{keV}$ ), while keeping the X-ray source size close to the laser optical focal spot size $(10-20 \mu \mathrm{m})$, with a $800 \mathrm{~nm}, 30 \mathrm{TW}$ laser $(10 \mathrm{~Hz}$ repetition rate and $30 \mathrm{fs}$ pulses $)$ by adequately controlling the optical contrast ratio at the fundamental $800 \mathrm{~nm}$ wavelength [72]. A K-alpha X-ray source generated with $100 \mathrm{TW}$ laser systems (30 J, $320 \mathrm{fs}$ ) has been used, with a high conversion efficiency of 9x10 $0^{-5}$ at $\sim 4.5$ $\mathrm{keV}$ (Ti K-alpha lines), to study interaction of X-rays with matter [73]. Laser based hard X-ray $(10-50 \mathrm{keV})$ sources including K-alpha have been recently studied with higher repetition rate laser systems $(100 \mathrm{~Hz}-10 \mathrm{KHz}$ range $)$ with wavelength in the IR and the mid IR, in view of potential applications in medical imaging with phase contrast techniques [74] or to be used for probing ultrafast matter dynamics [75-77]. The control of the optical contrast ratio is key in generation of X-rays from a very small spot size and with high conversion efficiency from optical to X-ray energy $\left(\mathrm{E}_{\mathrm{X}} / \mathrm{E}_{\mathrm{L}}\right)$. The highest conversion efficiency reported (around $2 \times 10^{-4}$ for Mo K-alpha) is similar to the one obtained with X-ray tubes and corresponds to the optimum conversion from electron distribution into X-ray K-alpha lines. The final throughput of a laser based K-alpha X-ray source for phase contrast imaging will thus be related to the 
repetition rate (in the few hundreds $\mathrm{Hz}$ to $\mathrm{kHz}$ range) and the average power (in the few hundreds $\mathrm{W}$ to $\mathrm{kW}$ range) of the driving system.

\subsection{Imaging with ultrafast laser K-alpha X-ray sources}

There is a large body of data showing that LPP-based K-alpha X-ray sources can be applied for biomedical imaging. The Lund's group [84-86] reported the first application of the LPP-based K-alpha X-ray source to radiological imaging. Barty et al. and the Lund group also demonstrated the possibility of time-gated medical imaging using a laser-based Xray source $[87,88]$. We have demonstrated the feasibility of CPA ultra-fast lasers for mammography [69-71] and digital energy subtraction angiography (DESA) [5]. Our group also showed the potential of laser-based X-ray sources for in line phase contrast imaging with $\mathrm{K} \alpha$ radiation around $25 \mathrm{keV}$ [89-91]. The typical laser shots number required to create an image of a mouse with good signal-to-noise ratio was around 6000 at $\mathrm{Ag} \mathrm{K}$-alpha emission lines, $0.2 \mathrm{~J}$ per shot, the source-to-detector distance of $2 \mathrm{~m}$ and a conversion efficiency of $2 \times 10^{-5}$ [90]. The acquisition time was thus around 10 min per image using a $10 \mathrm{~Hz}$ repetition rate laser system working at $400 \mathrm{~nm}$ fundamental wavelength. Higher conversion efficiency in the K-alpha line $\left(2 \times 10^{-4}\right)$, as obtained with our $10 \mathrm{~Hz}, 100 \mathrm{TW}$ peak power laser system [72] working at longer wavelength of $800 \mathrm{~nm}$, helps reducing the acquisition time. Another strategy to shorten the image acquisition time is to use high repetition rate lasers (in the $\mathrm{kHz}$ range) and increase the average laser power. The high repetition rate laser technology may allow the development of lower cost laser-based X-ray sources [92]. Such higher repetition rate X-ray sources have been used for small avascular tumor imaging [93] and differential phase contrast and tomography of mouse model [94].

The LPP-based K-alpha X-ray source has very attractive radiological features including small effective spot size and easy control of the X-ray spectrum via target change, and holds great promise for diagnostic and biomedical imaging using X-rays in the $15-50 \mathrm{keV}$ range. The LPP-based K-alpha X-ray sources require lasers with very high average power (in $\mathrm{kW}$ range) on the target in order to perform clinical X-ray computed tomography and other imaging examinations in an acceptable time frame. Such high average power is beyond reach of current ultrafast laser technology. However, it is a subject of intense research, particularly in Europe, aiming at development of ultrafast lasers based on new materials allowing higher repetition rates. These new emerging technologies will allow achieving hundreds or even thousands of Watts of average power with diode pumped pump laser systems in the femtosecond and picosecond pulse duration range. Therefore, we expect that such high-power lasers will become available in the near future and LPP-based $\mathrm{K}$-alpha X-ray sources will be applied in preclinical and biomedical imaging and eventually will be applied to imaging of human patients.

\section{LASER WAKEFIELD X-RAY BASED IMAGING}

The other major class of laser-based X-ray source that has been developed over the past ten years, is based on the phenomenon of electron acceleration by the laser wakefield. It can be divided into two main mechanisms, the Betatron and the Inverse Compton emissions. They are able to generate X-rays in the $50 \mathrm{keV}-\mathrm{few} \mathrm{MeV}$ range. Such X-ray sources have been created using sub-hundred femtosecond high-energy Ti:sapphire laser systems. Tajima \& Dawson first described in 1979 [95] the acceleration of electrons through the interaction of a laser with plasma. The coupling between plasma and an electromagnetic wave gives rise to a very large variety of interactions, including parametric instabilities and particle acceleration. They all strongly depend on the laser and plasmas parameters. The laser wakefield acceleration (LWFA) of electrons takes place when a short laser pulse interacts with a gas jet. The laser pulse induces a plasma wave, which originates from the charge separation between electrons and ions in the generated plasma. At low optical power density (not exceeding $10^{17} \mathrm{~W} / \mathrm{cm}^{2}$ ), the electron density perturbation follows a linear behavior along the laser pulse propagation axis and the associated electric field is low. At higher intensities (above $10^{18} \mathrm{~W} / \mathrm{cm}^{2}$ ), the electron density perturbation is no longer linear and very sharp and strong electric field gradients are generated. In this regime, electrons are accelerated in the ion cavity (bubble) produced in the wake of the laser pulse by the ponderomotive force $[96,97]$. With ultrafast laser parameters available today, the electric field can reach $600 \mathrm{GV} / \mathrm{m}$ with a cavity radius around $7 \mu \mathrm{m}$ for a gas density of $1 \times 10^{19} \mathrm{~cm}^{-3}$. The first reports of the generation of quasi mono-energetic electrons beams by the LWFA process were published in 2004 [98-100].

Our group studied LWFA since 2009 in close collaboration with Laboratoire d'Optique Appliquée (LOA, Ecole Polytechnique) in France [100]. In 2012, we reached remarkable performance (in terms of spectrum and stability) for the LWFA by controlling the injection of the electrons via generation of a sharp density transition [101]. We demonstrated 
with this technique that it was possible to generate, with a $80 \mathrm{TW}$ laser (30fs), quasi-mono-energetic electron beams with energy over $400 \mathrm{MeV}$ and an average charge close to $100 \mathrm{pC}$ [101]. We are reporting here results obtained with another injection method based on colliding beams [102-104], to improve the characteristics and stability of the accelerated electron beam. In previous experiments with this technique [103,104], the generation of quasi-mono-energetic electrons beams was reported with energies of $14 \mathrm{MeV}(22 \mathrm{pC})$ [103] and $117 \mathrm{MeV}(19 \mathrm{pC})$ [104]. We used two counter propagating colliding pulses. The main beam $(2.7 \mathrm{~J}, 30 \mathrm{fs}$ pulse) is focused with a $1.5 \mathrm{~m}$ parabola in an $18 \mu \mathrm{m}$ spot (FWHM) at an intensity of $1.25 \times 10^{19} \mathrm{~W} / \mathrm{cm}^{2}$. The colliding pulse $(100 \mathrm{~mJ}, 30 \mathrm{fs})$ is propagating along an axis tilted by $5^{\circ}$ from the main beam axis and is focused with a $50 \mathrm{~cm}$ parabola in a $16 \mu \mathrm{m}$ spot at an intensity of $4 \times 10^{17} \mathrm{~W} / \mathrm{cm}^{2}$. The target is a supersonic He gas jet produced with a $5 \mathrm{~mm}$ nozzle. When the electron density is $8 \times 10^{18} \mathrm{~cm}^{-3}$ the main beam alone is producing by self-injection an electron beam having a broad spectrum with a maximum energy around $240 \mathrm{MeV}$ and a charge around $160 \mathrm{pC}$. At a density of $5 \times 10^{18} \mathrm{~cm}^{-3}$ the main beam alone is not accelerating electrons and no electrons are detected. With the two beams, temporally synchronized, at this low density we systematically detect a quasi-monoenergetic electron beam with peak energy around $270 \mathrm{MeV}$, energy spread of $30 \mathrm{MeV}$ (FWHM) and an average charge close to $30 \mathrm{pC}$.

\subsection{Betatron radiation from Laser Wakefield Acceleration}

Ultrafast laser wakefield accelerated electrons perform wiggler-like oscillations creating a very bright micrometer-sized highly directional emission x-ray source [105-107]. The first demonstration of the generation of Betatron radiation was done in 2004 [105], but the radiation was emitted in the soft X-ray range. We did in 2010 the first demonstration of generation of laser-based betatron radiation in the hard X-ray range $(>10 \mathrm{keV})$ [108]. This source is characterized by a micrometric source size (lower than the size of the "bubble" generated by the laser wakefield), a divergence of a few tens of mrad, a broad spectrum of synchrotron radiation with photon energies in the $10 \mathrm{keV}-100 \mathrm{keV}$ range, and a pulse duration similar to the femtosecond laser pulse duration (i.e. $<100 \mathrm{fs}$ ). These sources require a laser with high peak power $(>100 \mathrm{TW})$ in order to reach the relativistic regime needed to accelerate electrons to a sufficiently high energy $(\sim$ $300 \mathrm{MeV}$ ) for the production of radiation in the betatron range at desired X-ray energy ( $>10 \mathrm{keV})$. Scaling laws have been proposed for optimization of electron acceleration in LWFA [109-111] and for optimization of the Betatron radiation as a function of the laser and the gas jet parameters for different acceleration regimes [107,112]. The betatron emission has been predominantly studied and optimized with ultrashort laser pulses ( $<100 \mathrm{fs})$. The development of new high average power laser technology with pulse durations in the 1-5 ps regime and experimental programs in High Energy Physics with high energy laser systems (TITAN, PETAL) with pulse durations in the 500 fs range are further motivating research aiming at optimization of generation of betatron radiation [113] through electron acceleration in regimes different from the LWFA including Self Modulated Laser Wakefield Acceleration (SMLWFA) [114-117] or the Direct Laser Acceleration (DLA) [113,118-120].

\subsection{Inverse Compton scattering radiation from Laser Wakefield Acceleration}

In the Inverse Compton Scattering (ICS) process two laser beams are used. The first beam creates a beam of energetic electrons in a gas jet by LWFA mechanism, while the second, fully synchronized, beam collides with the electron beam resulting in X-rays emission. The X-ray radiation is produced at the point of the collision and is emitted in the small solid angle in the direction of the electron beam. A third laser pulse at low energy could be used to control the electrons injection in the laser wakefield plasma accelerator. It allows generation of a quasi-monoenergetic electron beam with tunable energy, which is required (monochromatic electrons and an undulator mode) to produce monochromatic X-rays. $\mathrm{X}$-rays in the $\mathrm{MeV}$ energy range can be produced with electrons in the few hundred MeV energy range. There are only a few experiments in the inverse Compton Scattering regime associated with LWFA. The most recent work [121] demonstrated the generation of X-rays in the MeV range when a $100 \mathrm{TW}$ laser pulse interacted with a $200 \mathrm{MeV}$ electron beam generated by LWFA using another similar laser beam from the same laser. The measured brightness was around $10^{7}$ photons with an energy distribution peaked at $1 \mathrm{MeV}$, with the source size is $5 \mu \mathrm{m}$ and the divergence of $10 \mathrm{mrad}$. Both Betatron and Inverse Compton emission X-rays were emitted but the conversion efficiency of the Betatron and Inverse Compton processes seemed to be low. Several designs of all optical MeV energy X-ray sources have been recently published $[122,123]$.

\subsection{The Betatron Beam Line with the SPACE laser at INRS}

From 2010 to 2014, we developed and operated an X-ray betatron beamline with $100 \mathrm{TW}$ on target $(3 \mathrm{~J}$ in $30 \mathrm{fs}$ at $1 \mathrm{~Hz}$ repetition rate, limited by the target system). Under these conditions, we generated $2.2 \times 10^{8}$ photons $/ 0: 1 \%$ bandwidth/sr/shot at $10 \mathrm{keV}$ [108]. A fit to a synchrotron distribution provided a critical energy $\mathrm{E}_{\mathrm{c}}=12.3 \mathrm{keV}$ with 2.5 
$\mathrm{keV}$ precision. The total number of photons over the whole spectrum, obtained from the synchrotron fit distribution, was $\mathrm{N}=10^{9}$ with a confidence interval $\mathrm{N}=8.0-13.5 \times 10^{8}$ (using the measured solid angle). The X-ray spot size was measured using a knife-edge technique and was found to be $1.7 \mu \mathrm{m}$ (FWHM) [108]. The X-ray beam intensity distributions (10$20 \mathrm{keV}$ X-ray range) measured for various nozzle lengths $(3 \mathrm{~mm}, 5 \mathrm{~mm}$ and $7 \mathrm{~mm})$ are shown in Figure 1 . The laser-based betatron X-ray source provides a very attractive alternative to conventional microfocal X-ray tube, as well as to synchrotron-based X-ray source due to its high brilliance, microscopic effective size, and cone beam geometry offering a large field-of-view for imaging. In 2016, our X-ray betatron beamline was upgraded and is now operated with 250 TW on target ( $6 \mathrm{~J}$ in $20 \mathrm{fs}$ at $2.5 \mathrm{~Hz}$ repetition rate). The X-ray source design parameters are as follows: $10^{9}$ photons $/ 0: 1 \%$ bandwidth/sr/shot at $40 \mathrm{keV}$, a critical energy between 40 and $70 \mathrm{keV}$, effective X-ray source size of $1 \mu \mathrm{m}$, a divergence between 10 and 50 mrads (FWHM), an X-ray beam pointing stability and an X-ray energy stability in the $2 \%$ rms range. Such improvements in our betatron beam line parameters are not only the result of the laser beam energy and intensity increase on the target but are also related to the reduction of the laser beam imperfections. Our recent 3D PIC calculations with the CALDER code [124] demonstrate that the laser beam quality plays major role in the LWFA performance [125]. Control of the phase and intensity distribution along the laser beam propagation axis inside the target has been introduced in the upgraded betatron beamline in order to fully optimize the LWFA parameters (photon number, spectrum, stability and pointing) and maximize generation of betatron radiation.
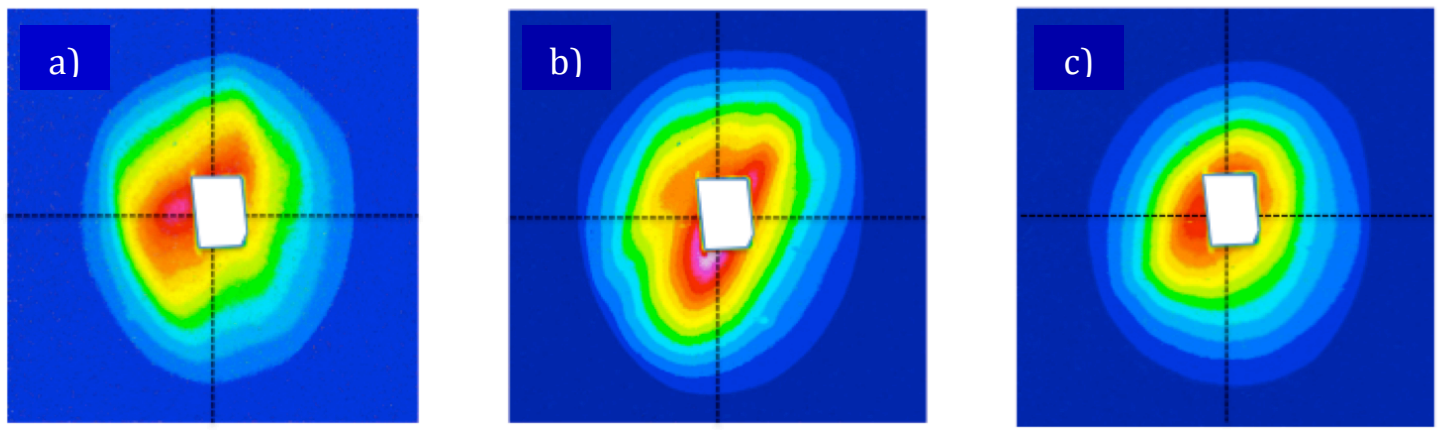

Figure 1: Measured radiation intensity in the 10-20keV X-ray range at $100 \mathrm{TW}$ on target for $3 \mathrm{~mm}(\mathrm{a}), 5 \mathrm{~mm}$ (b) and $7 \mathrm{~mm}$ (c) nozzle lengths. The white region depicts an aperture in the imaging system to simultaneously record the spectrum [108].

\subsection{High Throughput imaging with ultrafast laser Betatron radiation}

In 2011, at INRS [126], we demonstrated that one phase contrast hard X-ray image could be produced in one laser shot (with $100 \mathrm{TW}$ on target, $2.2 \times 10^{8}$ photons $/ 0: 1 \%$ bandwidth/sr/shot emitted at $10 \mathrm{keV}$, and with a synchrotron spectrum characterized by a critical energy $\mathrm{Ec}=12.3 \mathrm{keV}$ ) with a reasonable signal to noise ratio (around 6 for an image obtained in one shot). Similar results were and simultaneously obtained at U. of Michigan [127]. This provides opening of a new route for high throughput 2D imaging and fast 3D imaging of various biological objects and animal models with very high resolution and low dose [128,129].

Betatron X-ray sources have a unique X-ray pulse duration characteristics because they are as short as the driving optical laser pulses. It offers extraordinary opportunity for femtosecond molecular imaging. The INRS betatron beamline has been used recently to demonstrate the feasibility of femtosecond time-resolved X-ray absorption spectroscopy of Warm Dense Plasmas (at solid density with temperature in the $25 \mathrm{eV}$ range) in the $1-2 \mathrm{keV}$ range, with 30 fs temporal resolution [130].

Using the betatron X-ray source working at $1 \mathrm{~Hz}$ repetition rate one can thus perform ultra-high resolution $(\sim 1 \mu \mathrm{m})$ tomography of complex biological objects and animal models in less than 10 minutes or one can screen (via $2 \mathrm{D}$ images) 18,000 objects in a 5 hours run. Laser-based betatron hard X-ray sources have the potential to overcome many critical barriers in the imaging of animal models of diseases and might lead to paradigm shift in a wide range of applications including preclinical and eventually human patients' imaging. 


\subsection{Imaging with Inverse Compton scattering radiation X-ray source}

A monochromatic and tunable X-ray source, created with Inverse Compton Scattering (ICS), could improve quality of spectral X-ray imaging, as compared to existing dual energy micro-CT that rely on polychromatic X-ray sources [23-25]. $\mathrm{X}$-ray phase contrast tomography has been demonstrated with X-rays (at $21 \mathrm{keV}$ ) generated through Inverse Compton Scattering on electron beam $(20-45 \mathrm{MeV})$ generated with a small electron storage ring [131]. These results indicate that ICS, which provides a good level of coherence and monochromaticity, is promising. However, the combination between a laser and an electron ring remains complex and gives today access to limited range of X-ray energies. All-optical ICS systems combine tunable X-ray energy over large range and a narrow spectral band [132]. Such characteristics are very desirable in imaging.

\section{CONCLUSIONS}

K-alpha X-ray sources have characteristics very suitable for radiologic and biomedical applications. They are simple, compact, monochromatic, easily tunable, and with small effective X-ray focal spot size $(\sim 10 \mu \mathrm{m})$. However, due to low conversion efficiency and isotropic emission, very high average power (KW range) laser systems are needed. Such lasers are not presently available but they are under development in many countries and they might become available in the near future.

Another approach to generation of tunable and bright monochromatic X-ray source is through the Inverse Compton Scattering (ICS) process. However, such source is complex and to-date no imaging has been reported for all-optical ICS systems.

Betatron X-ray source provides an interesting compromise in terms of X-ray source performance and system complexity. As of now, it is therefore a unique alternative, with $1 \mu \mathrm{m}$ effective source size, high brilliance and energy range up to hundreds of $\mathrm{keV}$ for very high throughput and high-resolution imaging. Although the spectral shape in betatron emission is broad (synchrotron type), X-ray phase contrast imaging and dual energy imaging can be performed. Because of highly directional emission the average laser energy in the tens of Watts range is sufficient for biomedical and preclinical imaging, while hundreds of Watts is sufficient for radiological applications. Such lasers are already available and practical imaging applications of betatron X-ray source are within reach for the first time.

\section{ACKNOWLEGMENTS}

The authors would like to thank J. Ferry, A. Lifschitz, V. Malka, A. Rousse, K. Ta Phuoc (LOA), J.L. Bourgade, X. Davoine (CEA-DAM), J. Maltais, S. Payeur, A. Saraf (INRS) and E. Hallin (GIFS) for stimulating discussions, their help at different stages during experiments and data interpretation. This work was funded by NSERC, The Programme de Chaire de Recherche du Canada, The CFI and FQRNT.

\section{REFERENCES}

[1] CA Combs. Fluorescence Microscopy: A Concise Guide to Current Imaging Methods. Current protocols in neuroscience / editorial board, Jacqueline N Crawley [et al]. 0 2:Unit2.1-Unit2.1.2. (2010)

[2] Legres LG et al, The Laser Technology: New Trends in Biol- ogy and Medicine. Journal of Modern Physics, 5, 267-279 (2014).

[3] Q. Peng at al, Report on Progress in Physics, 71, 1, (2008)

[4] R. L. Ehman et al, Radiology, 244, 12 (2007)

[5] J.C. Kieffer et al, Future of laser-based X-ray sources for medical imaging, Appl. Phys. B 74, S75 (2002)

[6] D. Regulla et al, Radiation protection Dosimetry, 114, 11 (2005)

[7] F. Mettler et al, Radiology, 248, 254 (2008)

[8] A Oppelt Imaging Systems for Medical Diagnostics: Fundamentals, Technical Solutions and Applications for Systems Applying Ionizing Radiation, Nuclear Magnetic Resonance and Ultrasound, Edition 2 John Wiley \& 
Sons, (2011); Diagnostic Radiology Physics - a handbook for teachers and students, 2014 International Atomic Energy Agency, Vienna, (2014).

[9] EA Rafferty et al, Breast Cancer Screening Using Tomosynthesis and Digital Mammography in Dense and Nondense Breasts. Jama. 2016;315:1784-1786 (2016).

[10] Assessing and improving the interpretation of breast images, workshop summary. The national Academy Press, Washington DC (2015)

[11] L Li et al, A Review of Imaging Techniques for Plant Phenotyping. Sensors (Basel, Switzerland). 14:2007820111 (2014).

[12] A. Pierret et al, Agronomie 23, 471 (2003)

[13]L. Young et al, J. Experimental Botany, 58, 2513 (2007)

[14] M. Mascher et al, The Plant Journal 76, 718 (2013)

[15] J. Hsieh, Computed tomography, SPIE Press (2003)

[16] S.R. Cherry, Phys. Med. Biol. 49, R13 (2004)

[17] M. Khalil et al, Int. J. Mol. Imaging 796025 doi:10.1155/2011/796025 (2011)

[18] P.T. Callagham, Clarendon Press, Oxford (1991)

[19] P. Glover et al, Rep. Prog. Phys. 65, 1489 (2002)

[20] A. Fenster et al, Phys. Med. Biol.46, R67 (2001)

[21] N. Deshpande et al, Clin. Radiology 65, 567 (2010)

[22] D. Holdsworth et al, Trends in Biotechnology, 20, 534 (2002)

[23] C.T. Badea et al, Medical Imaging SPIE proceedings, Vol. 6913, 691342 (2008)

[24] C. T. Badea et al, Medical Imaging SPIE proceedings, Vol. 7961, 79611X (2011)

[25] D. P. Clark et al, Phys. Med. Biol. 58, 1683 (2013)

[26] Z. Starosolski et al, Sci. Rep. 5, 10178 (2015)

[27] M. Zhang et al, Med. Phys. 41, 031907 (2014)

[28] W. Cai et al, J. of Nucl. Med.49, 114S (2008)

[29] W. Koba et al, The Am. J. of Pathology 182, 319 (2013)

[30] R. Yao et al, J. Nucl. Med. Technology 40, 157 (2012)

[31] H. Wehrl et al, Eur. J. Nucl. Med. Mol. Imaging 36, S56 (2009)

[32] D. Gabor, “A New Microscopic Principles”, Nature 161, No. 4098, 777 (1948).

[33] D. Gabor, "Microscopy by Reconstructed Wavefronts”, Proc. Roy. Soc. (London) A 197, 454 (1949).

[34] A. Snigirev et al, Rev. Sci. Instrum. 66, 5486 (1995)

[35] S. W. Wilkins et al, Nature, 384, 335 (1996)

[36] A. Momose, Jpn. J. Appl. Phys. 44, 6355 (2005)

[37] Z. Wang et al, $7^{\text {th }}$ Medical applications of Synchrotron Radiation Workshop (MASR 2012), published by IOP publishing for Sissa Medialab (2013)

[38] Y. Zhao et al, Proc. Nat. Acad. Sci. USA, 109, 18290 (2012)

[39] F. Arfelli et al, Radiology, 215, 286 (2000)

[40] P. Coan et al, J. Phys. D, 46, 494007 (2013)

[41] A. Momose et al, Nature Med. 2, 473 (1996)

[42] T. Thüring et al, Appl. Phys. Lett. 103, 091105 (2013)

[43] R. A. Lewis, Phys. Med. Biol.49, 3573 (2004)

[44] E. Lombi, J. Sisini, Plant Soil, 320, 1 (2009)

[45] L. W. Young et al, J. Exp. Botany, 58, 2513 (2007)

[46] R. A. Lewis, Phys. Med. Biol. 49, 3573 (2004)

[47] H. Rieger, X-ray visions. SPIE's OE Magazine, March, 26-28 (2001)

[48] J. Duderstadt and Moses GA, Inertial Confinement fusion, Wiley-Interscience Publ. (1992).

[49] P.J. Mallozi et al, J. Appl. Phys. 45,1891 (1974).

[50] J.C. Kieffer et al, Phys. Rev. Lett. 50, 1054 (1983)

[51] Strickland D and Mourou G, Opt. Commun. 56, 219 (1985)

[52] Perry MD and Mourou G, Science 264917 (1994)

[53] Barty CPJ, Laser Focus World June 93-106, (1996)

[54] S. Backus et al, Rev. Sci. Instrum. 69, 1207 (1998)

[55] W. Sibbet et al, Opt. Express, 20, 6989 (2012).

[56] P. Russbueldt et al, Opt. Express, 17, 12230 (2009) 
[57] J. Mende et al, SPIE proceedings, 7193, 71931V-1 (2009)

[58] A. Maksimchuk et al, Phys. Rev. Lett. 84, 4108 (2000)

[59] R. Snavely et al, Phys. Rev. Lett. 85, 2945 (2000)

[60] S. Wilks et al, Phys. of Plasmas, 8, 542 (2001)

[61] Y. Yin et al, Phys. of Plasmas, 18, 063103 (2011)

[62] S. Mangles et al, Nature 431, 535 (2004)

[63] C. Geddes et al, Nature 431, 538 (2004)

[64] J. Faure et al, Nature 431, 541 (2004)

[65] A. Gopal et al, Phys. Rev. Lett. 111, 074802 (2013)

[66] F. Albert et al, LLNL-Proc-666251 (2015)

[67] C. Danson et al, High Power Laser Science and Engineering, 3, 1 (2015)

[68] Laser acceleration of Electrons, protons and ions; and medical applications of laser generated secondary sources of radiation and particle; SPIE Proceedings Vol. 8079, W. Leemans, E. Esarey, S. Hooker, K. Ledingham eds. (2011)

[69] A. Krol et al, SPIE Proceedings, Vol. 3157, 0277-786X (1997)

[70] A. Krol, A. Ikhlef, J.C. Kieffer, D.A. Bassano, C. Chamberlain, Z. Jiang, H. Pepin, S. Prassad: Med. Phys. 24, 725 (1997)

[71] J. Yu, Z. Jiang, J.C. Kieffer, A. Krol: IEEE J. of Selected Topics in Quant. Electron. 5, 911 (1999)

[72] S. Fourmaux et al, Appl. Phys. B 122:162, doi:10.1007/s00340-016-6442-8 (2016)

[73] A. Lévy et al, Appl. Phys. Lett. 96, 151114 (2010)

[74] C. Serbanescu et al, SPIE proceedings, 7451, 745115. doi:10.1117/12.826646 (2009)

[75] T. Lee et al, Proceedings of SPIE, Vol 4978, 77 (2003)

[76] L. Miaja-Avila et al, Structural Dynamics 2, 024301 (2015)

[77] F. Zamponi et al, Appl. Phys. A, doi:10.1007/s00339-009-5171-9 (2009)

[78] J. Weisshaupt et al, Nature photonics, doi:10.1038/NPHOTON.2014.256 (2014)

[79] Z. Zhang et al, Opt. Express, 19, 4560 (2011)

[80] L. Chen et al, Phys. Rev. Lett., 100, 045004 (2008)

[81] L. Chen et al, Phys. of Plasmas 11, 4439 (2004)

[82] J. Yu et al, Phys. of Plasmas, 6, 1318 (1999).

[83] K. Huang et al, Rev. Sci. Instrum. 85, 113304 (2014)

[84] K. Herrlin, G. Svahn, C. Olsson, H. Pettersson, C. Tillman, A. Persson, C.G. Wahlstrom, S. Svanberg: Radiology 189, 65 (1993)

[85] C. Tillman, A. Persson, C.G. Wahlstrom, S. Svanberg, K. Herrlin: Appl. Phys. B 61, 333 (1995)

[86] C. Tillman, I. Mercer, S. Svanberg, K. Herrlin: JOSA B 134, 209 (1996)

[87] C.P.J. Barty, et al, SPIE Proc. 2523, 286 (1995)

[88] C.L. Gordon III, et al, Opt. Lett. 20, 1056 (1995)

[89] R. Toth et al, Rev. Sci. Instrum.76, 083701 (2005)

[90] R. Toth et al, Phys. of Plasmas, 14, 053506 (2007)

[91] A. Krol et al, Proceedings SPIE, Vol 6510 (2007).

[92] J.A. Chakera et al, Appl. Phys. Lett. 93, 261501 (2008)

[93] Y. Nesterets et al, Proc. Soc. Photo Opt. Instrum. Eng. 6913, 69133 (2008)

[94] C.M. Laperle et al, Appl. Phys. Lett., 91, 173901 (2007)

[95] T. Tajima and J. Dawson, Phys. Rev. Lett. 43, 267 (1979);

[96] A. Pukhov and J. Meyer-ter-Vehn, Appl. Phys. B, 74355 (2002)

[97] V. Malka, Phys. of Plasmas, 19, 055501 (2012)

[98] S. P. D. Mangles, et al, Nature 431, 535 (2004).

[99] C. G. R. Geddes, et al, Nature 431, 538 (2004).

[100] J. Faure, Yet al, Nature 431, 541 (2004).

[101] S. Fourmaux et al, Appl. Phys. Lett.101, 111106 (2012);

[102] E. Esarey, et al, Phys. Rev. Lett. 72, 1224 (1994)

[103] H. Kotaki et al, Phys. Plasmas 11, 3296 (2004)

[104] J. Faure, et al, Nature, 444, 737 (2006)

[105] A. Rousse et al, Phys. Rev. Lett. 93, 135005 (2004)

[106] K. Ta Phuoc et al, Phys. Rev. Lett. 97, 225002 (2006) 
[107] S. Corde et al, Rev. Mod. Phys. 85, 1 (2013)

[108] S. Fourmaux et al, New Journal of Physics, 13, 033017 (2011)

[109]C.B. Schroeder et al, Phys. Rev. ST Accel. Beams 13, 101301 (2010)

[110]B. Pollok et al, Phys. Rev. Lett. 107, 045001 (2011)

[111]K. Nakajima et al, High Power laser Science \& Engineering 3, e10 doi:10.1017/hpl.2015.5 (2015)

[112]A. Pukhov et al, Phys. of Plasmas, 6, 2847 (1999)

[113]F. Albert et al, Plasma Phys. Control. Fusion 56, 084015 (2014)

[114]C. A. Coverdale et al., Phys. Rev. Lett. 74, 4659 (1995).

[115]D. Umstadter et al, Science 273, 472 (1996).

[116]N. Hafz et al, Int. J. of Modern Phys. B 21, 398 (2007)

[117]N. Lemos et al, Plasma Phys. Control. Fusion 58, 034018 (2016)

[118] T.W. Huang et al, Phys. Rev. E, 93, 063203 (2016)

[119]X. Zhang et al, Phys. Rev. Lett., 114, doi: 10.1103/PhysRevLett.114.184801 (2015)

[120]B. Liu et al, Phys. Rev. Lett., 110, 045002 (2013)

[121] Chen et al, Phys. Rev. Lett. 110, 155003 (2013)

[122] K. Nakajima, High Power laser Science and Engineering, Vol. 2, e31, (2014)

[123] Y. Ping et al, Laser and Particle beams 31, 607 (2013)

[124] E. Lefebvre et al., Nuclear Fusion 43, 629 (2003).

[125] J. Ferri et al, Sci. Rep. 6, 27846 doi:10.1038/srep27846 (2016)

[126]S. Fourmaux et al, Opt. Lett. 36, 2426 (2011)

[127]S. Kneip et al, Appl. Phys. Lett. 99, 093701 (2011)

[128]J.M. Cole et al, Sci. Rep. 5, 13244, doi:10.1038/srep13244 (2015)

[129]J. Wenz et al, Nature Communications 6:7568 doi:10.1038/ncomms8568 (2015)

[130]M. Mo et al, Rev. Sci. Instrum. 84, 123106 (2013)

[131]E. Eggl et al, PNAS Vol. 1125567 (2015)

[132]S. Banerjee et al, Nucler Instrum. And Meth. In Phys. Res. B 350, 106 (2015) 